\title{
Cultura e inteligencia musical mediante la investigación como estrategia pedagógica en educación básica ${ }^{1}$
}

\section{$\overline{\text { Culture and musical intelligence through research }}$ as a pedagogical strategy in basic education}

DOI: http://dx.doi.org/10.17981/cultedusoc.9.3.2018.96

Artículo de investigación. Fecha de recepción: 15/06/2018. Fecha de aceptación: 27/11/2018

\author{
Nurys Caballero-Sanjuán² y Carolina Curiel-Amastha ${ }^{3}$ \\ IED Francisco de Paula Santander (Colombia) \\ nuryecaballero@hotmail.com
}

Para citar este artículo:

Caballero-Sanjuán, N. y Curiel-Amastha, C. (2018). Cultura e inteligencia musical mediante la investigación como estrategia pedagógica en educación básica. Cultura. Educación y Sociedad 9(3), 813-822. DOI: http://dx.doi.org/10.17981/cultedusoc.9.3.2018.96

\section{Resumen}

La educación ha presentado cambios significativos en los últimos años por lo cual desde el rol docente es necesario implementar estrategias dinamizadoras que estén orientadas a mejorar las competencias de los estudiantes, favoreciendo el proceso enseñanzaaprendizaje. El estudio tuvo como objetivo promover la cultura e inteligencia musical mediante la investigación como estrategia pedagógica (IEP) en educación básica. Con un diseño metodológico de tipo cualitativo bajo el modelo de investigación Acción Participativa (IAP), las técnicas de recolección de datos utilizadas fueron la observación participante y el diario de campo. En cuanto a la población beneficiaria se realizó un muestro aleatorio estratificado utilizando como criterio de inclusión el interés por la cultura, música e investigación, la muestra estuvo conformada por cien (100) estudiantes con edades entre los seis (6) y doce (12) años, de todos los niveles de primaria de la IED Francisco de Paula Santander ubicada en el municipio de Fundación Magdalena. Los resultados dejaron en evidencia que a través de la cultura e inteligencia musical permite reforzar la memoria con tareas cognitivas, de igual forma posibilitan la asimilación de contenidos pertinentes a los desarrollados en clases de una manera eficaz y amena con un sentido de creación de un ambiente idóneo, y llevando a la vez al resto de la comunidad estudiantil incentivando a la cooperación entre los estudiantes

Palabras clave: cultura, inteligencia musical, investigación como estrategia pedagógica.

\section{Abstract}

The education has presented significant changes in the last years so from the teaching role it is necessary to implement dynamic strategies that are oriented to improve the students' competences, favoring the teaching-learning process. The objective of the study was to promote culture and musical intelligence through Research as a Pedagogical Strategy (IEP) in basic education. With a qualitative methodological design under the participatory action research (IAP) model, the data collection techniques used were the participant observation and the field diary. Regarding the beneficiary population, a stratified random sampling was carried out, using as an inclusion criterion the interest for culture, music and research, the sample consisted of one hundred (100) students with ages between six (6) and twelve (12) years, of all the primary levels of the IED Francisco de Paula Santander located in the Municipality of Magdalena Foundation. The results showed that through the culture and musical intelligence techniques are carried out that reinforce the memory, cognitive tasks for the learning, ways to solve the communicative problems, as well as activities that allow an assimilation of the contents in an efficient way, thanks to the creation of a favorable environment, companionship and cooperation among students.

Keywords: culture, musical intelligence, research as a pedagogical strategy.

\footnotetext{
1 Este artículo deriva del Programa de Fortalecimiento de la Cultura Ciudadana y Democrática CT+I a través de la IEP apoyada en TIC en el Departamento de Magdalena: CICLON
}

2 Líder del grupo de investigación "Juventud investigadora".

3 Docentes de la institución educativa Francisco de Paula Santander, sede principal, del grupo de investigación "Juventud investigadora".

- The author; licensee Universidad de la Costa - CUC

Cultura, Educación y Sociedad vol. 9 no. 3, pp. 813-822. Diciembre, 2018

Barranquilla. ISSN 2389-7724 Online 


\section{Introducción}

A lo largo de la historia, el concepto de inteligencia ha ido evolucionando, esto demuestra la capacidad de manipular símbolos, de procesar información y de resolver problemas, otras concepciones defienden el concepto de la inteligencia humana, no por los procesos y operaciones mentales, sino más bien por la capacidad de actuar que posee el ser humano, dirigir el comportamiento, conocimiento de la realidad y la capacidad de crear nuevas posibilidades.

Sin ninguna duda, hoy por hoy, la música es arte, es fuente de inspiración y alegría en todos los escenarios, ya que por medio de ella se puede sensibilizar para producir y pensar en términos de ritmos, tonos o timbres de los sonidos. Es por ello que las personas se inclinan por tocar instrumentos musicales, cantar, silbar, escuchar música, componer melodías o atender a sonidos ambientales, son personas innovadoras, capaces de expresar y canalizar sus emociones y sentimientos y con una gran capacidad de desarrollar las matemáticas. De igual manera, posibilita en transportarnos a diferentes épocas de la vida, a momentos de alegría dolor, pasión, tristeza; toca cada uno de los sentidos y trasmite lo que muchos desean expresar. Desde la Prehistoria, la música es fundamental en todas las culturas. Sin embargo, se ha minimizado su valor e importancia en la existencia de cada persona y en la posibilidad que brinda para el desarrollo intelectual, cultural y social.

En efecto, es necesario incentivar, reconocer y hacer trascender la música, como forma de expresión, como medio de adquirir destrezas para el aprendizaje y como transformación de la realidad social $\mathrm{y}$ cultural en la que actualmente estamos inmersos. La música tiene esa particularidad de cambiar nuestro estado de ánimo si lo deseamos. Desde el punto de vista de la neurociencia, sabemos que la música es muy poderosa a la hora de activar cada una de las estructuras emocionales en el cerebro.

Dadas las condiciones que anteceden, la institución educativa Francisco de Paula Santander está presentando falencias en el proceso de aprendizaje que influye en el rendimiento académico de los estudiantes, es el poco trabajo que se genera con ellos a partir del desarrollo de su inteligencia, como resultado a esto se consideró trabajar en estrategias culturales para desarrollar la inteligencia musical en los estudiantes del nivel primaria y secundaria de educación básica, con esto se busca impactar en la inteligencia en general del estudiante que le permita aplicar los mecanismos adecuados para promover la práctica de la cultura e inteligencia múltiple para optimizar su proceso cognitivo, social, psicomotor, y emocional.

Teniendo en cuenta el apartado anterior si no se realiza un plan significativo sobre las condiciones educativas y sociales en el continuo trabajo de incentivar la inteligencia que le permita desempeñarse no solo en las áreas artísticas si no en otras áreas, como consecuencia a esto incidirá sobre su educación, y en sus diferentes contextos sociales y familiares y por consiguiente en el mantenimiento de sus funciones motoras, auditivas, visuales, perceptuales.

En forma general en algunos estamentos de servicios educativos que brindan atención a los niños, niñas y jóvenes del nivel secundaria no priorizan el incentivo de la inteligencia del alumno en el hecho de cultivar su cultura e inteligencia musical como elemento integrador de la sociedad escolar y, por consiguiente, no sé aplican las técnicas necesarias para subsanar estas dificultades y de esta forma poder mejorar el proceso enseñanza y aprendizaje. 
A esto se suma, la falta de programas que involucren ejercicios dirigidos para fomentar la cultura e inteligencia musical de los estudiantes a través de actividades motivadoras para fomentar el lenguaje corporal y su relación con el entorno.

Los movimientos de coordinación, óculo manual, y psicomotriz gruesa y fina, son características que han sido observadas para determinar el grado de dificultad que presentan los niños, teniendo en cuenta su ubicación en el espacio y contexto no debe hacer diferencia o preferencia su inteligencia dominante, además, se interrelaciona directamente con todas las áreas del desarrollo, a través de sus dimensiones cognitivas, afectivas y motrices.

La creatividad es más que una actividad motora ya que ayuda a afianzar la cultura e inteligencia múltiple de los niños, niñas y jóvenes cuyo punto de referencia es el cuerpo, el ritmo, actividades motrices. Esta etapa es la mejor instancia para estimular la inteligencia como proceso dinámico que tiende a poner al individuo en relación con el ambiente para que adquiera independencia y su aprendizaje sea más eficaz. Los estudiantes hacia los cuales se dirigirá el siguiente proyecto son los estudiantes de la IED Francisco de Paula Santander en sus diferentes grados académicos, teniendo en cuenta para este proyecto una muestra que nos permita elaborar las actividades y mostrar los resultados.

En ese mismo sentido, una organización cultural e inteligencia musical apoya el funcionamiento de la memoria dado que la información que llega al hemisferio izquierdo necesita también en globalizar el lado derecho, cuando se asocian ambas informaciones, activan la función y adquiere una unidad cognitiva en un sentido de memorizar en el corto y a largo plazo, ello requiere de suma atención en poder interpretar la información en referencia a captar los distintos sonidos e imitarlos, además de tener sensibilidad al ritmo, discriminar las cualidades del sonido, escuchar cantar con un sentido de interpretación canciones y obras, así como una predisposición a tocar instrumentos. En todas estas funciones incide mediante una funcionalidad hemisférica y su integración a través del cuerpo calloso. En los procesos cerebrales para el ritmo, las poesías y el toque de instrumentos musicales influye en las diferentes funciones de los lóbulos cerebrales, el cuerpo calloso y el sistema límbico.

El estudio pretende destacar la importancia de las promoción de cultura e inteligencia musical mediante la IEP en los estudiantes de todos los niveles de educación básica de la Institución Educativa Departamental Francisco de Paula Santander del municipio de FundaciónMagdalena, mediante la implementación de actividades relacionadas con la cultura e inteligencia musical que involucren estrategias orientadas a mejorar sus competencias en el proceso cognitivo, emocional y social, afianzando el aprendizaje de manera transversal a las áreas del currículo.

Para esta investigación, se parte de comprender que las capacidades de los niños y niñas en edades tempranas deben ser estimuladas adecuadamente para que a lo largo de su vida puedan enfrentar diversas situaciones apropiadamente con el orgullo y la satisfacción de poner en juego estas habilidades encaminados hacia el éxito. Teniendo en cuenta que el individuo es un ser con potencialidades a desarrollar, se debe brindar un contexto adecuado donde se interactúa con la naturaleza, que se reconozca así mismo y a los demás, potencializando cada una de las Inteligencias Múltiples en cualquier ámbito de aprendizaje.

Sumado a lo anterior, el generar este tipo de investigaciones, se pretende aportar herramientas metodológicas a 
las docentes que apoyan la intervención en el aula y que redundan en el beneficio colectivo al facilitar el aprendizaje en ambientes diseñados para estimular el desarrollo motor de niños, es decir, que tengan en cuenta las edades, capacidades, manejo de espacios, recursos y formas de abordar la planeación didáctica desde la lectura del contexto. La importancia de la presente investigación, radica en la necesidad de transformación del docente que trabaja en las aulas de primera infancia, teniendo en cuenta que su quehacer diario impacta a la población, al facilitar o entorpecer el desarrollo al máximo de sus capacidades: físicas, intelectuales, expresión musical, corporal y apropiación del medio.

\section{Cultura e inteligencia musical mediante la IEP: un impacto socio-académico}

Cultura es el conocimiento, el arte, las creencias, la ley moral, las costumbres y todos los hábitos y habilidades adquiridos por el hombre no sólo en familia, sino también al ser parte de una sociedad como miembro de ella. Por su parte, la palabra supone una habilidad en la composición, función y consideración de patrones musicales, abarcando la capacidad de reconocer y componer tonos musicales y ritmos esto enmarcados en resolver problemas y dificultades. En sentido amplio la inteligencia es la capacidad de asimilar, guardar, elaborar información y utilizarla donde a través de ella podemos mejorar nuestra atención y concentración, además tienen competencias para discriminar velozmente los sonidos mediante a combinaciones musicales.

Por otro lado, la inteligencia musical es la capacidad de producir, apreciar el ritmo el tono y el timbre de los sonidos valorando las distintas formas de expresividad musical. Esto implica crear, reconocer y producir música, incluye la sensibilidad para percibir e identificar las formas musicales y transformarlas. $\mathrm{Al}$ adquirir la destreza de esta inteligencia se logra apreciar una estructura y el ritmo de la música se genera además sensibilidad para producir sonido, melodía, ritmo y tono. (Cortacáns y Almudena, 2011; Ramírez, Chacón y El Kadi (2018). La música hace parte del área de artística A través de ella se busca que el niño participe activamente en las actividades y obtenga de ellas experiencias positivas que le ayuden en su desarrollo.

Sánchez y Andrade (2014) afirma que las personas tenemos distintas inteligencias que podemos desarrollar con la adecuada práctica o ejercicio. De acuerdo a lo anterior, las Inteligencias Múltiples se pueden llevar a cabo en diferentes combinaciones por medio de la práctica o ejercicios, ya que desarrollamos unas más que otras y hay que fortalecerlas, para así aprovechar las habilidades innatas que posee cada ser.

Para Gardner (1983), la inteligencia es una capacidad que se desarrolla a lo largo de la vida y esta variabilidad se da en la relación con entornos de aprendizaje enriquecidos, interacciones y vivencias que originan procesos de asimilación, acomodación y construcción de redes de conocimientos y/o puentes de relación que permiten llevar a cabo un proceso de estimulación desde la Inteligencia que se encuentra más desarrollada para aportar a las que necesite fortalecer. Desde esta perspectiva, el desarrollo de las Inteligencias surge a partir de las capacidades y el conocimiento que adquiere cada persona con base en su adecuada 
estimulación durante todo su ciclo vital, cabe señalar que para Gardner la inteligencia también es "la capacidad para resolver problemas o para elabora productos que son de gran valor para un determinado contexto [...]" por lo que esto hace referencia a la importancia que tienen la inteligencia musical dentro del ámbito cotidiano (Sánchez y Andrade, 2014; Ramírez Molina Reynier Israel y Ampudia Sjogreen Daniel Esteban, 2018).

A propósito, Torres (2004) indica que "es importante adaptar la lúdica a las necesidades, intereses y propósitos del nivel educativo. En ese sentido el docente de educación inicial debe desarrollar la actividad lúdica como estrategia pedagógica respondiendo satisfactoriamente a la formación integral del niño y la niña". Desde esta perspectiva, los responsables de la educación del presente siglo deberán tener horizontes claramente definidos en cuanto a los fines que ésta persigue, con el propósito de transitar de la mano mediante normatividad y dentro del ejercicio de autonomía, articular los contenidos programáticos de los planes de estudios.

En ese orden ideas, Castillo (2016) y Ramírez, Avendaño, Aleman, Lizarazo, Ramírez y Cardona (2018), manifiestan que el enseñar al niño a leer y escribir es un proceso que no se debería iniciar antes de los seis o siete años, esto debido en gran parte a que, los niños, niñas antes de esa edad trabajan principalmente, con los tres dedos de la mano que representa un dominio donde estos le servirán para coger el lápiz, en ese sentido, son ejercicios que en marcan una condición de orden y concentración sensoriales donde el niño se enfoca en la utilización del hemisferio izquierdo al derecho lo que le va servir en el aprendizaje utilizando mecanismos inherente a la formas amplias, enriqueciendo su desarrollo y potenciando habilidades como ver, oír y representar patrones melódicos, proporcionando una memoria musical y componentes perceptivos. .

En opinión de Waichman (2000) "es imprescindible la modernización del sistema educativo para considerar al estudiante como un ser integral, participativo, de manera tal que lo lúdico deje de ser exclusivo del tiempo de ocio $\mathrm{y}$ se incorpore al tiempo efectivo de y para el trabajo escolar" Pascual (2002), postula que la música es de suma importancia en el desarrollo físico y mental en las personas; es por eso que en su método plantea la incursión de la enseñanza musical desde los primeros años de educación (sería la educación pre-escolar) y este encuentra su real valor en el ejercicio activo y la participación de actividades musicales.

En este mismo orden de ideas Calderón (2015), expone que es tarea de los docentes es descubrir las aptitudes de los niños, para así desarrollar su creatividad, imaginación y su expresividad; además ver la música como un medio de expresión en el ser humano, este a su vez responden con interés a una gran variedad de sonidos, incluyendo la voz humana, los sonidos del entorno que son organizados en un modelo significativo para los niños que parte de los 3 elementos fundamentales de esta (ritmo, armonía y melodía), pues el ritmo nos acompaña en el mayor parte de nuestros quehaceres. Es 
por esto que se ve la importancia que los docentes quienes desde preescolar deben involucrar la música utilizarla como estrategia dinamizadora y así facilitar los procesos de aprendizaje.

Por su parte las estrategias pedagógicas son todas las acciones realizadas por el docente, con el fin de facilitar la formación y el aprendizaje de los estudiantes. "Componen los escenarios curriculares de organización de las actividades formativas y de la interacción del proceso enseñanza y aprendizaje donde se logran conocimientos, valores, prácticas, procedimientos y problemas propios del campo de formación" (Bravo, 2008).

Las estrategias pedagógicas suministran invaluables alternativas de formación que se desperdician por desconocimiento y por la falta de planeación pedagógica, lo que genera monotonía que influye negativamente en el aprendizaje. Sobre la base de las consideraciones anteriores una estrategia pedagógica es una guía de acción, en el sentido de que orienta a la obtención de ciertos resultados. (Cortacáns y Almudena, 2011). Desde la experiencia docente las estrategias son sensibles a la modificación constantemente a partir de los propios cambios que se vayan operando en el objeto de estudio. Estas responden a los objetivos definidos para los estudiantes en cada nivel de educación.

En la actualidad, existen propuestas metodológicas basadas en enfoques investigativos, entendidos como la forma de materializar lo que se hace y como se implementa donde surgen infinidad de propuestas metodológicas que toman varias propuestas metodo- lógicas; es el caso de las pedagogías basadas o fundadas en investigación, las cuales se desarrollan en diferentes contextos y asumen variados caminos en coherencia con los paradigmas y corrientes en los cuales se inscriben. Dentro de esta teoría basada en investigación se encuentra la enseñanza por descubrimiento y el Programa Ondas, con su propuesta de la Investigación como estrategia Pedagógica (IEP), como una perspectiva que se desarrolla en ese enfoque y toma elementos de la pedagogía critico-liberadora del enfoque socio-cultural, (Mejía y Manjarrés, 2010; Herrera, Guerrero y Ramírez, 2018).

En ese sentido, considerar la investigación como estrategia pedagógica implica recrear un espacio de inclusión, donde se respete y se viva la diversidad haciendo evidentes los canales de la creatividad del docente, para convertir las preguntas de los grupos de aprendizaje colaborativo conformados por niños, niñas y adolescentes en una investigación, llevándolos a tomar un rol activo en el proceso de aprendizaje mediante la construcción de su propia conocimiento, potenciando así, las habilidades y destrezas de los estudiantes, convirtiendo la educación en un vehículo de capacitación, de oportunidades y de recreación (Camargo, 2015).

Es un ejercicio que va a requerir a todo educador, reconocerse y construirse como actor en estas nuevas condiciones, lo cual va a exigir un replanteamiento de las formas anteriores y la consabida modificación de su nueva condición, así como de las transformaciones educativas, las cuales desarro- 
llan su quehacer mediante la subjetividad y los escenarios de su acción se convierten en campos resemantizados $\mathrm{y}$, por lo tanto, en disputa para construir un proyecto de autonomía o de simple modernización al servicio de la colectividad en la sociedad. Es allí donde el educador pone en escena su capacidad humana al servicio de intereses precisos y concretos (Cetrulo, 2001; Ramírez y Hugueth, 2017).

Dicho en otras palabras, se requiere reconocer la importancia de la investigación en la sociedad, a la vez que se develan los intereses presentes. En su práctica forja una mirada crítica, que en coherencia con los tiempos presentes se ve obligada no solo a la denuncia, sino también a apropiarse de ella como una realidad muy importante en esta época para construir propuestas que a la vez que promuevan modernizar y transformar ciudadanos ejemplares para la sociedad, con un sentido integral en todos los ámbitos de este mundo tan complejo, mediante nuevas mediaciones científico-tecnológicas de este tiempo para hacer concreta la construcción sociedades más democráticas, justas y humanas, así como la necesidad de un planteamiento sobre ella en cualquier proyecto emancipador.

En ese mismo sentido, la idea de aprendizaje es retomada también de este enfoque y reelaborada para Vigotsky: "El aprendizaje es un aspecto universal y necesario del proceso de desarrollo culturalmente organizado y específicamente humanos de las funciones psicológicas"; es decir, este, organizado pedagógicamente, se convierte en desarrollo mental y sigue generando procesos evolutivos que no se podrían dar sin él. Es allí donde las relaciones entre el sujeto y el mediador son activas y recíprocas, en la internalización se da el conocimiento. En ese sentido, el aprendizaje es una actividad propia diferenciada, por ello no se puede hacer equivalente a desarrollo.

\section{Metodología}

\section{Diseño}

Se realizó un estudio de tipo cualitativo, utilizando el modelo de investigación por acción, desde un diseño descriptivo. La investigación cualitativa se fundamenta en una perspectiva interpretativa centrada en el entendimiento del significado de las acciones de los participantes, a su vez postula que la "realidad" se define a través de las interpretaciones de los mismos en la investigación respecto a sus propias realidades (Hernández, Fernández, y Baptista, 2010).

La investigación acción plantea una producción de conocimiento basado en la reflexión de los participantes o sujetos de la investigación, investiga con los actores y no a los actores sociales. Se desarrolla la producción de un conocimiento integrador y contextual que nace del trabajo del grupo sobre una realidad determinada en un proceso colaborativo.

Los estudios con un diseño descriptivo están encaminados a especificar las características, perfiles y propiedades de personas, grupos, comunidades, procesos, objetos o cualquier otro fenómeno que sea sometido a un análisis, donde el corte transversal hace referencia a la temporalidad de la investigación, la cual será en un espacio de tiempo corto, buscando solucionar de forma rápida el problema (Hernández, Fernández y Baptista, 2010). 


\section{Participantes}

La unidad de análisis estuvo conformada por cien (100) estudiantes de la IED Francisco de Paula Santander, sede principal de todos los grados de básica primaria y básica segundaria. Ubicados en el $\mathrm{Mu}$ nicipio de Fundación, Magdalena. Cabe destacar que los participantes cumplieron con los requisitos de diligenciamiento del consentimiento informado.

\section{Técnicas e instrumentos}

Para la recolección de datos se utilizó la encuesta, esta se aplicó a los estudiantes, es decir, estuvo conformada por 10 Ítems de preguntas cerradas, cuyos resultados se tabularon y procesaron estadísticamente para determinar la situación en que se encuentra el objeto de la investigación. En cuanto a la población beneficiaria se realizó un muestro aleatorio estratificado utilizando como criterio de inclusión el interés por una cultura, música e investigación.

\section{Resultados}

De acuerdo a las técnicas utilizadas y a los datos obtenidos en los resultados al finalizar el proyecto se puede concluir que a través de la cultura e inteligencia musical se llevan a cabo técnicas que refuerzan la memoria, tareas cognitivas para el aprendizaje, una forma de concientizar al claustro que es importante que para mejorar la calidad de enseñanza se debe trabajar con todas las inteligencias, en ese sentido para sacar lo mejor de los estudiantes la inteligencia musical ha de ocupar un lugar que corresponda dentro de la malla curricular, esta a su vez debe fomentar conceptos tendientes en aprender técnicas referidas en las distintas asignaturas, inventando ritmos y melodías sistematizando las actuaciones en cualquier escenario de la cotidiani- dad posibilitando una asimilación de los contenidos en referencia de una manera eficaz, gracias a la creación de un ambiente favorable, al compañerismo y a la cooperación entre los alumnos.

Además, los contenidos programáticos pueden ser llevados al aula con el fin de entrenar a los alumnos en su uso y capacitarles de los medios necesarios para enfrentarse al reto de aprender de su cultura e inteligencia musical de una manera amena y significativa. Por su parte, la Institución Educativa, dentro de su plan de estudios no contempla proyectos relacionados con la cultura e inteligencia musical aplicada a las actividades extracurriculares.

Partiendo del proceso de indagación, no se evidencian programas de actividades de cultural que respondan a las necesidades encontradas en la población estudiantil en cuanto al desarrollo de actividades para favorecer su inteligencia musical. Y el aprendizaje de la cultura municipal e inteligencia musical ocurre de acuerdo con el contexto donde el niño desarrolla su vivencia, ya que es coordinado, gradual y simultaneo en la enseñanza aprendizaje. Los estudiantes han incentivado a través de distintas actividades a las sedes de la Institución como medio para lograr impactar aún más, $\mathrm{y}$ crecer en cuanto al número de estudiantes integradores del proyecto, por medio de esto han logrado participar en diferentes festivales en distintas regiones y municipios aledaños. De acuerdo con los resultados arrojados por esta propuesta la investigadora del proyecto recomienda lo siguiente:

a. Diseñar un plan de actividades de cultura que identifican al pueblo permanente para que los niños, niñas y adolescentes de la Institución desarrollen su Inteligencia musical y por consiguiente mejoren su aprendizaje. 
b. Que los docentes y directivos docentes se apropien de las necesidades de los estudiantes en el sentido de orientarlos apropiadamente sobre las actividades que deben desarrollar en el aula de clases.

c. Involucrar a los padres y madres de familia en el desarrollo de la propuesta, haciéndolos partícipes de la misma como agentes de apoyo a las diversas actividades programadas.

d. Los estamentos docentes y directivos docentes deben liderar proyectos para darle solución a la problemática en la identificación cultural.

e. Participación activa en las actividades culturales programadas en la Institución para así demostrar sus talentos.

f. Utilizan con más propiedad las TIC en la institución, ya saben indagar mejor sus tareas con estas herramientas.

Seguidamente, es importante llevar a cabo estrategias pedagógicas teniendo presente la diversidad y el desarrollo de la infancia, puesto que todos los niños no poseen las mismas capacidades de aprendizaje, cómo se manifestó en este proyecto, tomando en cuenta las características más relevantes de la población. Logrando con esto un puente de relación desde la Inteligencia Musical, con las dos Inteligencias a estimular: Kinestésica Corporal y Visual Espacial.

\section{Conclusiones}

La cultura e inteligencia musical mediante la investigación como estrategia pedagógica en educación básica, es una oportunidad para comunicarse a través de movimientos, permitiendo favorecer su sentido auditivo aportando al reconocimiento del entorno por medio, de sonidos y ruidos que lo conforma. Mediante la música a la hora de cantar incrementa el vocabulario, proporcionando fluidez en cuanto a su oralidad, se produce un mayor desenvolvimiento en su entorno, obteniendo así una seguridad de sí mismos.
Todo lo anterior basado en la infancia, en vista de que en esta etapa los niños disfrutan del libre movimiento, les gusta la imitación de sonidos.

Con base en lo anterior se logra un aprendizaje propio, se generó un puente de relación logrando estimular la inteligencia visual espacial y kinestésica corporal, puesto que en la música se permiten movimientos corporales logrando un desplazamiento e interacción con su espacio. Por otro lado, junto con la musicalidad no solo se pueden lograr los aspectos ya mencionados, si no, también está puede ir encaminada hacia la integración con otros factores, logrando así un aporte más amplio.

\section{Referencias}

Araque, F. y Suárez, O. (2017). Equidad Ética-Jurídica de la Ciencia, para la Emancipación del Conocimiento y los Saberes. JURÍDICAS CUC, 13(1). 97120. DOI: http://dx.doi. org/10.17981/ juridcuc.13.1.2017.5

Bravo, H. (2008). Estrategias pedagógicas. Córdoba. Universidad del Sinú.

Calderón, A. (2015). La música como estrategia dinamizadora para facilitar los procesos de aprendizaje en la educación inicial. [Tesis]. Universidad del Tolima, Ibague.

Camargo, C. (2015). La investigación como estrategia pedagógica en la Guajira, desde una perspectiva de la inclusividad y diversidad como nuevo paradigma para el desarrollo de habilidades, destrezas y competencias. Educación y ciudad, (29), 149-162.

Castillo, A. (2016). La afectividad para el desarrollo de las macro destrezas en el área de lengua y literatura en los niños y niñas de segundo año de educación básica de la unidad educativa "Amelia Gallegos Díaz", del cantón Riobamba, provincia de Chimborazo. [Bachelor's thesis]. Riobamba, UNACH. 
Cetrulo, R. (2001). Alternativas para una acción transformadora: Educación popular, ciencias y política. Montevideo: Ediciones Trilce-Instituto del Hombre.

Cortacáns, C. y Almudena, M. (2011). Inteligencias múltiples en acción. Barcelona: Colegio Montserrat.

Gardner, H. (1993). Estructuras de la mente. Recuperado de http://educreate.iac a $\quad t$. com/Maestros/Howard_Gardner_-_Estructuras_de_la_mente.pdf

Herrera, B., Guerrero, H. y Ramírez, R. (2018). Investigación como estrategia pedagógica: Una mirada desde la educación, escuela y transformación de la comunidad global. Barranquilla: Editorial Universitaria de la Costa..

Hernández, R., Fernández, C. y Baptista, P. (2010). Metodología de la investigación. México, D.F.: Editorial Mc Graw Hill.

Mariño, G. (2010). El diálogo en la educación de jóvenes y adultos. Dos propuestas pedagógicas para implementarlo. En, El taller dialógico/la recuperación de experiencias laborales. Bogotá, D.C: OEI.

Mejía, M. y Manjarrés M. (2010). Las pedagogías fundadas en la investigación. Búsquedas en la reconfiguración de la educación. Revista Internacional Magisterio 42(1). 16-26.

Pascual, P. (2002). Didáctica de la música para primaria. El método Kodaly.

Ramírez, R., Chacón, H. y El Kadi Janbeih, O. (2018). Gestión estratégica del talento humano en las PYMES. Medellín: Editorial Corporación CIMTED.
Ramírez, R. y Hugueth, A. (2017). Modelo de comunicación productiva para las organizaciones de salud pública en Venezuela. Revista Opción. 33(83). 305-335. Disponible en http://produccioncientificaluz.org/index.php/opcion/article/ view/23118

Ramírez, R. y Ampudia, D. (2018). Factores de Competitividad Empresarial en el Sector Comercial. Revista Electrónica de Ciencia y Tecnología del Instituto Universitario de Tecnología de Maracaibo (RECITIUTM). 4(1). 16-32. Disponible en http://recitiutm.iutm.edu.ve/ index.php/recitiutm/article/view/130

Ramírez, R., Avendaño, I., Aleman, L., Lizarazo, C., Ramírez, R. y Cardona, Y. (2018). Principles of social responsibility for the strategic management of the talent human public health organizations. Revista Espacios, 39(37). 22-27. Disponible en http://www.revistaespacios.com/a18v39n37/18393722.html

Ramírez, R., Chacón, H. y Valencia, K. (2018). Gestión del talento humano como estrategia organizacional en las Pequeñas y Medianas Empresas. Revista CI$C A G, 16(1):$ 20-42. Disponible en http:// ojs.urbe.edu/index.php/cicag/article/ view/2929

Segura, E. (2016). Información, estabilidad y complejidad de aprendizaje en memorias asociativas. International Journal of Management Sciences and Operations Research, 1(1), 49-53. Recuperado de http://ijmsoridi.com/index.php/ijmsor/article/view/77

Torres, L. (2004). Tres enfoques teóricopráctico. México, D.F.: Trillas.

Waichman, A. (2000). Herramientas de pensamiento. España: Siglo XXI. 